\title{
Multinucleated HIV-1-infected T cells exhibit unique migration patterns and frequent virus- dependent cell-cell interactions
}

Menelaos Symeonides $^{1}{ }^{\text {, Emily E. Whitaker }}{ }^{2,1}$, Markus Thali ${ }^{2,1}$ ${ }^{1}$ Department of Microbiology and Molecular Genetics, University of Vermont, USA. ${ }^{2}$ Cellular, Molecular, and Biomedical Sciences Graduate Program, University of Vermont, USA

\section{Abstract Text}

HIV-1-infected T cells found within secondary lymphoid tissue, such as lymph nodes and the spleen, can fuse with uninfected $T$ cells to form small syncytia, the majority of which have only 2 nuclei. This observation, documented by several groups (Mempel, Chen, Mothes) in the BLT humanized mouse model, was recapitulated in our lab using simple in vitro 3D culture systems (Symeonides et al., 2015). Syncytia could be found at relatively high frequencies (up to $20 \%$ of all infected $T$ cells in the lymph node), which was surprising given the wide array of fusion-inhibitory mechanisms that operate during cell-to-cell HIV-1 transmission. Additionally, small syncytia were induced (both in vivo and in vitro) by CCR5-tropic strains, including transmitted-founder strains, raising the possibility that syncytia are a fundamental facet of early HIV-1 infection, and that they may thus play an active role in HIV-1 spread (Compton \& Schwartz, 2017). We took advantage of the in vitro 3D culture system we established to dissect facets of HIV-1 spread and document the role and behavior of these syncytia compared to mononucleated infected cells (and uninfected cells) in the same environment.

To facilitate large-volume, long-term, and low-phototoxicity live imaging of HIV-1-infected T cells in 3D collagen hydrogels, we established light sheet fluorescence microscopy in our lab using the single-sided inverted selective plane illumination microscopy (iSPIM) 
platform originally developed by the Shroff lab (NIBIB, NIH). We conducted imaging experiments under cell culture conditions $\left(37^{\circ} \mathrm{C}\right.$, $5 \% \mathrm{CO}_{2}, 95 \%$ humidity; Okolab) using up to three excitation channels (488 nm, $561 \mathrm{~nm}, 640 \mathrm{~nm}$ ) and 40x illumination and detection objectives. Stage scans on the order of $1 \mathrm{~mm}$ were performed with time intervals ranging $40 \mathrm{sec}-4 \mathrm{~min}$ for total timelapse durations of up to $12 \mathrm{~h}$. Use of fluorescent reporter viruses (kind gifts from Benjamin Chen) allowed identification of infected cells, and nuclei were labeled using SiR-DNA. Imaging datasets were deskewed in SlideBook 6 (Intelligent Imaging Innovations), and subsequently imported into Vision4D 3.3 (Arivis AG) for drift correction, segmentation, and automated 3D tracking.

Tracks were manually curated and corrected, and finally exported as per-cell/per-timepoint XYZ coordinates for migration pattern analyses (including track speed, acceleration, straightness, displacement ratio, outreach ratio, arrest coefficient, and tortuosity). In parallel, cells of interest were manually analyzed to document infecteduninfected cell-cell contacts (virological synapses) and virus transfer events. Our preliminary analyses indicate that syncytia form virusdependent cell-cell contacts more frequently, but nevertheless migrate more persistently and exhibit increased environment scanning behavior, compared to mononucleated infected cells. These unique behaviors likely endow syncytia with increased virus spreading ability, and may explain the presence of syncytia as a significant subpopulation of HIV-1-infected T cells in vivo. 